\title{
Gastrointestinal and Liver Manifestations of COVID-19
}

\author{
Sunil Taneja ${ }^{1}$ Rohit Mehtani ${ }^{1}$ Yogesh Kumar Chawla ${ }^{2}$ \\ ${ }^{1}$ Department of Hepatology, Postgraduate Institute of Medical \\ Education \& Research, Chandigarh, India \\ 2Department of Gastroenterology, Kalinga Institute of Medical \\ Sciences, Bhuvneshwar, India
}

Address for correspondence Yogesh Kumar Chawla, MBBS, MD, DM, Department of Gastroenterology, Kalinga Institute of Medical Sciences, Bhuvneshwar, Odisha 751024, India (e-mail: ykchawla@gmail.com).

Ann Natl Acad Med Sci (India):2020;2:91-98

\begin{abstract}
Keywords

- COVID-19

- gastrointestinal

- liver

- RNA $\beta$-coronavirus

- SARS- CoV-2

A novel Coronavirus, SARS-CoV-2 illness, has spread throughout the world after the first case was reported from Wuhan, China, in December 2019. This illness typically causes respiratory symptoms like fever, cough, and shortness of breath, although atypical presentation with gastrointestinal symptoms like abdominal pain, nausea, vomiting, or diarrhea are being increasingly reported. The viral RNA has been detected in saliva and stool of such patients, which raises concerns regarding the risk of transmission during gastrointestinal (Gl) endoscopy. Many patients also have liver involvement, with the most common manifestation being deranged liver function tests. This review highlights the symptomatology, mechanism, and histopathology findings of SARS-CoV-2 in Gl tract and liver. This review also focuses on implications of COVID-19 in patients afflicted with chronic liver disease and in patients undergoing liver transplantation.
\end{abstract}

\section{Introduction}

In December 2019, a novel Coronavirus named as severe acute respiratory syndrome coronavirus 2 (SARS-CoV-2) was first reported from Wuhan, China. ${ }^{1}$ It is a nonenveloped RNA $\beta$-coronavirus ${ }^{2}$ which has infected 4307287 people with more than 295000 deaths as of May 15, 2020. ${ }^{3}$ This outbreak is significantly bigger than the previous pandemics of SARS and Middle East respiratory syndrome (MERS), which were caused by different strains of coronavirus. While lungs are the primary organ of involvement in Coronavirus disease 2019 (COVID-19), liver, and gastrointestinal (GI) involvement is being increasingly reported in the emerging data from various centers across the globe.

\section{Information Source and Literature Search}

For this review article, we searched PubMed for articles published from January 1, 2020 using the keywords "COVID-19," "coronavirus," "severe acute respiratory syndrome coronavirus 2," "SARS-CoV-2," and "2019-nCoV." Also, publications on COVID-19 from various journals, including The Lancet, The New England Journal of Medicine, JAMA, BMJ, Gut, Gastroenterology, Journal of Hepatology, Hepatology,
Liver International, Hepatology International were screened, and relevant articles including preproof publications were included. Further articles were included after screening the reference list of included studies.

\section{GI Symptomatology}

The clinical presentation of COVID-19 patients is variable. The most common and typical symptoms reported from a multicenter study from China are fever (88.7\%) and cough (67.8\%). ${ }^{4}$ While GI symptoms are infrequent, nausea/vomiting is seen in around 5\% patients and diarrhea in 3.8\%. ${ }^{4}$ However, in the study by Fang et al, ${ }^{5} \mathrm{GI}$ involvement was reported in up to $79 \%$ of patients with predominant symptoms being anorexia, diarrhea, nausea/vomiting, abdominal pain, and GI bleed. The GI symptoms can also precede fever and dyspnea by 1-2 days in approximately $10 \%$ of patients. ${ }^{6}$ There is a recent meta-analysis of 57 studies $^{7}$ that has reported the prevalence of diarrhea in $7.7 \%$, nausea/vomiting in $7.8 \%$, and abdominal pain in $3.6 \%$ of patients. These symptoms of diarrhea, nausea/vomiting, and abdominal pain were reported more commonly from countries other than China. ${ }^{7}$ A large number of patients are being recognized in the asymptomatic stage or with atypical symptoms like pain abdomen, with abdominal 
CT scans suggestive of typical findings of COVID-19 in lung bases. ${ }^{8}$ Patients can even have GI symptoms in the absence of respiratory symptoms, as seen in $16 \%$ of patients in a study by Luo et al. ${ }^{9}$ Therefore, accurate history taking and documenting gastrointestinal complaints is essential to prevent missing out on the diagnosis of COVID-19 in patients who lack respiratory symptoms.

\section{GI Symptoms and Severity of COVID-19}

The presence of GI symptoms is associated with a more severe form of disease and was first reported by Henry et al. ${ }^{10}$ Abdominal pain and nausea/vomiting were associated with increased disease severity, while diarrhea had no such association. A pooled analysis of 10 studies having a total of 1989 confirmed COVID-19 patients revealed severe disease in around $30.1 \%$ of patients. In another study by Tian et al, ${ }^{11}$ patients with severe disease had more frequent GI symptoms as well (anorexia $66.7 \%$ vs. $30.4 \%$; abdominal pain $8.3 \%$ vs. $0 \%$. The patients with GI involvement are also more likely to have a longer duration of illness ( $33 \%$ vs. $22 \% ; p=0.048$ ) and test positive for COVID-19 (OR 1.7, 95\% CI 1.1-2.5) in one study. ${ }^{12}$

\section{Mechanism of GI-Tropism}

The entry of SARS-CoV2 into the host cell happens as a result of the interaction of envelope-anchored spike protein with angiotensin-converting enzyme 2 (ACE2), which is present on host cells. ${ }^{2}$ Spike protein of SARS-CoV-2 contains two subunits, S1 and S2. ${ }^{13}$ S1 is involved in virus attachment to the cell membrane, and S2 is involved in the fusion of cell membranes by using ACE2. ${ }^{14}$ Serine proteases, mainly Transmembrane protease serine 2 (TMPRSS2) is required for priming this process. ${ }^{15}$ Binding of SARS-CoV-2 with ACE2 may alter gut microbiota, interfere with innate immunity, and inhibit dietary tryptophan absorption, leading to diarrhea. ${ }^{16}$ High-ACE2 receptor staining in the cytoplasm of gastric, duodenal, and rectal epithelial cells of COVID-19 infected patients have been demonstrated, ${ }^{16}$ supporting viral entry and GI symptoms of diarrhea, abdominal pain, and nausea/ vomiting.

\section{Fecal-Oral Transmission of COVID-19-Is it Possible?}

The interest in GI involvement of SARS-CoV-2 was further augmented when viral RNA was detected by real-time reverse transcriptase polymerase chain reaction (rRT-PCR) in the stool sample of the first COVID-19 case in USA. ${ }^{17}$ This led to the proposition of the fecal-oral route of transmission (-Table 1). Viral RNA can be detected in stool in around 70\% of patients even after full resolution of symptoms. ${ }^{7}$ However, in spite of high-RNA concentration, viral isolation was not possible from stool samples, ${ }^{16}$ thereby questioning the infectivity of COVID-19 through the fecal-oral route. The presence of the live virus has also been detected in saliva by viral culture; however, the clinical significance of this has to be deciphered. ${ }^{18}$

\section{Histopathological Findings in GI Tract in COVID 19 Patients}

The histopathological findings in GI tract of COVID-19 patients are variable. The first autopsy report ${ }^{19}$ described segmental dilatation and stenosis of the small intestine, while histopathology was suggestive of degeneration, necrosis, and shedding of gastrointestinal mucosa in another patient. ${ }^{20}$ Endoscopic biopsies from the esophagus, stomach, duodenum, and rectum suggest that the esophagus shows occasional lymphocytic infiltrate, while lymphoplasmacytic infiltrate and interstitial edema was present in lamina propria of stomach, duodenum and rectum; ${ }^{16}$ however, there was no mucosal epithelial damage. Viral nucleocapsid protein was also demonstrated in gastric, duodenal, and rectal glandular epithelial cells in this case. In another autopsy study of two patients, ${ }^{21}$ no abnormalities in GI tract were noted except for increased visceral adipose tissue in both patients and gaseous distension of bowel loops in one patient.

\section{Liver Injury with COVID-19}

The most common liver injury documented in COVID-19 patients is abnormal liver function tests (LFTs), ${ }^{1,6,22,23}$ which is reported in approximately 14 to $53 \%$ patients, as shown in - Table 2. According to a recent meta-analysis, elevated AST and ALT are seen in 15\% of patients and elevated bilirubin in approximately $16.7 \%$ of patients, with a higher proportion of patients with deranged LFTs seen from countries other than China. ${ }^{7}$ Most cases are mild and transient, although elevated AST, ALT, ALP, or total bilirubin is associated with increased mortality. ${ }^{24}$ The pattern of liver injury is usually hepatocellular type, with AST being elevated more than ALT, and ALP largely remaining normal. ${ }^{24}$ Patients with GI symptoms are more likely to have liver dysfunction as well (17.57\% vs. $8.84 \%, p=0.035) .{ }^{25}$ The mechanism is unclear, and various factors are implicated like pre-existing chronic liver disease, drug toxicity, ischemic hepatitis secondary to hypotension and, rarely, direct injury by SARS-CoV-2.

Only one case of acute liver failure ${ }^{26}$ in a patient of SARS-CoV-2 has been described to date.

The patient was a 65-year-old hypertensive man who presented with typical respiratory signs and symptoms of COVID-19. He had elevated amino transaminases at admission. He required mechanical ventilation on day 2 of admission and was treated with ritonavir/lopinavir and interferon- $\beta$. However, therapy had to be stopped, as his aminotransferases started to increase from day 7 and reached a maximum by day 14 . The bilirubin went up to a maximum of $22.2 \mathrm{mg} / \mathrm{dl}$ by day 20 . The etiological workup for viral hepatitis $A, B, C$, and $E$ and autoimmune hepatitis were negative. At last follow-up (day 20), he was still critically ill, with multiorgan failure, and registered a model for end-stage liver disease (MELD) score of 40. 
Table 1 Summary of studies documenting viral RNA detection in feces

\begin{tabular}{|c|c|c|c|c|c|c|}
\hline $\begin{array}{l}\text { S. } \\
\text { No. }\end{array}$ & Authors & $\begin{array}{l}\text { Type of } \\
\text { Study }\end{array}$ & $\begin{array}{l}\text { No. of } \\
\text { patients }\end{array}$ & Gl symptoms & Sample & Results \\
\hline 1. & Jiang $X$ et $a^{54}$ & Case Report & 1 & Asymptomatic & Anal swab & Detected up to 42 days \\
\hline 2. & Siew $C \mathrm{Ng}$ et $\mathrm{a}^{55}$ & Case series & $\begin{array}{l}21 \text { cases and } \\
114 \text { healthy } \\
\text { individuals }\end{array}$ & NA & Feces & $\begin{array}{l}\text { Detected in all } 81 \text { stool samples } \\
\text { from } 21 \text { patients }\end{array}$ \\
\hline 3. & Wu et $a^{56}$ & $\begin{array}{l}\text { Observational } \\
\text { study }\end{array}$ & 132 & NA & $\begin{array}{l}\text { Anal swabs \& } \\
\text { feces }\end{array}$ & $\begin{array}{l}\text { RNA detected in } 9.8 \% \text { of stool } \\
\text { samples and } 10 \% \text { of anal swabs }\end{array}$ \\
\hline 4. & Wu et al ${ }^{57}$ & $\begin{array}{l}\text { Observational } \\
\text { study }\end{array}$ & 98 & $31 \%$ & Feces & $\begin{array}{l}55 \% \text { patients had positive fecal RNA. } \\
\text { Fecal samples remained positive for } \\
27.9 \pm 10.7 \text { days } \\
\text { While respiratory swabs were } \\
\text { positive for } 16.7 \pm 6.7 \text { days }\end{array}$ \\
\hline 5. & Chen et $a^{58}$ & $\begin{array}{l}\text { Retrospective } \\
\text { study }\end{array}$ & 42 & $19 \%$ & Feces & $\begin{array}{l}66.67 \% \text { patients had detectable RNA } \\
\text { in feces } \\
64.29 \% \text { patients remained positive } \\
\text { for RNA in feces for } 6-10 \text { days after } \\
\text { pharyngeal swabs were negative }\end{array}$ \\
\hline 6. & Chen et $a^{59}$ & Case report & 1 & NA & $\begin{array}{l}\text { Pharyngeal swab, } \\
\text { sputum and feces }\end{array}$ & $\begin{array}{l}\text { Pharyngeal swab and sputum } \\
\text { negative and fecal RT-PCR Positive }\end{array}$ \\
\hline 7. & Zhang et al ${ }^{60}$ & $\begin{array}{l}\text { Observational } \\
\text { study }\end{array}$ & $\begin{array}{l}39 \text { in } 1 \text { st investi- } \\
\text { gation and } 139 \\
\text { in } 2 \text { nd } \\
\text { investigation }\end{array}$ & NA & $\begin{array}{l}\text { Pharyngeal, oral } \\
\text { swab, anal swab } \\
\text { and serum }\end{array}$ & $\begin{array}{l}15 \text { patients had PCR positive after } \\
\text { treatment out of which } 4 \text { were anal } \\
\text { swab positive. On day } 5 \text {, anal swabs } \\
\text { were more positive than oral swabs } \\
\text { ( } 75 \% \text { vs. } 50 \%)\end{array}$ \\
\hline 8. & Yun et $a^{61}$ & $\begin{array}{l}\text { Retrospective } \\
\text { study }\end{array}$ & $\begin{array}{l}2510 \text { patients } \\
\text { screened in } \\
\text { fever clinic }\end{array}$ & NA & $\begin{array}{l}\text { Pharyngeal swab, } \\
\text { feces, serum }\end{array}$ & $\begin{array}{l}32(1.3 \%) \text { positive for RNA by } \\
\text { pharyngeal swab and eight patients } \\
\text { had positive RNA in feces }\end{array}$ \\
\hline 9. & Cheung et al ${ }^{62}$ & $\begin{array}{l}\text { Retrospective } \\
\text { data from } \\
\text { Hong-Kong } \\
\text { cohort \& } \\
\text { Systematic } \\
\text { review and } \\
\text { meta-analysis }\end{array}$ & $\begin{array}{l}59 \text { patients } \\
\text { Meta-analysis of } \\
60 \text { studies } \\
(n=4243)\end{array}$ & $\begin{array}{l}25.4 \% \\
17.6 \%\end{array}$ & Feces & $\begin{array}{l}15.3 \% \text { tested positive for RNA in } \\
\text { feces. } 38.5 \% \text { with diarrhea and } 8.7 \% \\
\text { without diarrhea had stool RNA } \\
\text { In meta-analysis, } 48.1 \% \text { stool } \\
\text { samples were positive for RNA. Of } \\
\text { these, } 70.3 \% \text { samples were collected } \\
\text { after respiratory samples were } \\
\text { negative }\end{array}$ \\
\hline 10. & Zhang et al ${ }^{63}$ & $\begin{array}{l}\text { Retrospective } \\
\text { study }\end{array}$ & 14 & None & $\begin{array}{l}\text { Oropharyngeal } \\
\text { swabs, feces }\end{array}$ & $\begin{array}{l}\text { Four out of } 62 \text { stool samples (6.5\%) } \\
\text { positive for viral RNA }\end{array}$ \\
\hline 11. & Xiao et al ${ }^{16}$ & $\begin{array}{l}\text { Observational } \\
\text { study }\end{array}$ & 73 & NA & $\begin{array}{l}\text { Serum, naso- } \\
\text { pharyngeal and } \\
\text { oropharyngeal } \\
\text { swabs, urine, } \\
\text { stool, endoscopic } \\
\text { biopsy }\end{array}$ & $\begin{array}{l}53.42 \% \text { had positive viral RNA in } \\
\text { feces } \\
23.29 \% \text { continued to have positive } \\
\text { fecal RNA after negative respiratory } \\
\text { swab }\end{array}$ \\
\hline 12. & Wei et $a^{64}$ & $\begin{array}{l}\text { Retrospective } \\
\text { study }\end{array}$ & 84 & $\begin{array}{l}31 \% \text { patients } \\
\text { had diarrhea }\end{array}$ & Feces & $\begin{array}{l}\text { Patients with diarrhea had longer } \\
\text { duration of fever/dyspnea }(p<0.05) \\
\text { Viral RNA in stool detected in } 69 \% \\
\text { with diarrhea and } 17 \% \text { without } \\
\text { diarrhea }(p<0.001)\end{array}$ \\
\hline
\end{tabular}

Abbreviation: NA, Not available.

Two cases of acute on chronic liver failure (ACLF) related to SARS-CoV-2 have been recently described. The first report ${ }^{27}$ of ACLF is by EASL-CLIF definition due to insult by SARS-CoV-2. The patient was a 56-year-old woman with alcohol-related decompensated cirrhosis and a history of upper GI bleed due to gastric varices.

The second $\operatorname{cas}^{28}$ describes a patient with nonalcoholic steatohepatitis (NASH) cirrhosis and ascites who was also admitted with complaints of nausea, vomiting, diarrhea, and anorexia without any respiratory symptoms that progressed to grade 2 ACLF for which liver transplantation was done at day 28 of admission.

\section{Postulated Mechanisms of Liver Injury}

\section{ACE2 in Liver Injury}

ACE2 receptors and TMPRSS2 are expressed in cholangiocytes and hepatocytes, and viral binding to these receptors may result in entry of SARS-CoV-2 in liver cells. ${ }^{29,30}$ However, only a small percentage of hepatocytes express ACE2 receptors $(\sim 2.6 \%){ }^{29}$ 


\begin{tabular}{|c|c|c|c|c|c|c|c|c|c|c|c|c|c|c|c|}
\hline 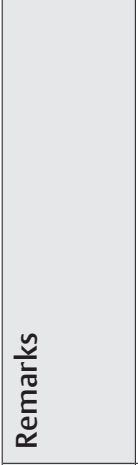 & 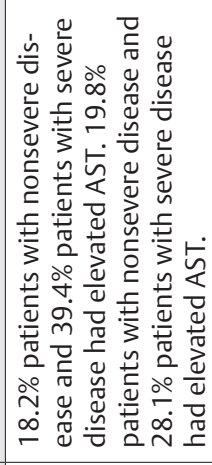 & 1 & 1 & 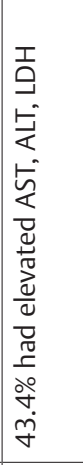 & 1 & 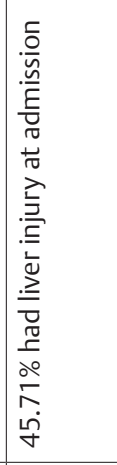 & 1 & 1 & 1 & 1 & 1 & 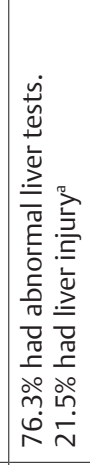 & 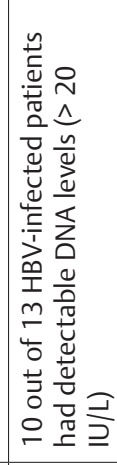 & 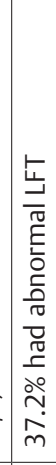 & \\
\hline 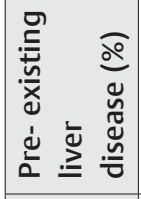 & $\bar{i}$ & $\S$ & 乏 & 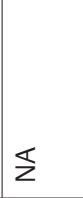 & $\begin{array}{l}\mathscr{c} \\
\text { Oे } \\
\end{array}$ & $\S$ & 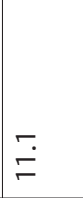 & $\stackrel{\stackrel{亠}{i}}{i}$ & 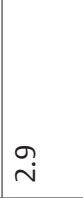 & ڤे & $\stackrel{m}{=}$ & $\begin{array}{l}\text { O̦ } \\
\text { in }\end{array}$ & 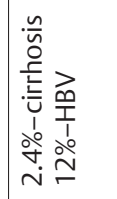 & $\bar{\varphi}$ & 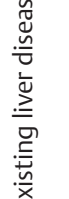 \\
\hline 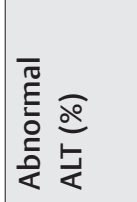 & $\frac{m}{\sim}$ & $\tilde{m}$ & $\Sigma$ & $\Sigma$ & $\Sigma$ & $\frac{\tilde{m}}{\stackrel{\sim}{\sim}}$ & $\stackrel{\infty}{\sim}$ & $\Sigma$ & $\Sigma$ & 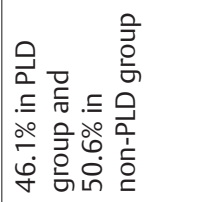 & $\Sigma$ & 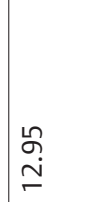 & $\Sigma$ & $\begin{array}{l}\underset{1}{\infty} \\
\stackrel{\infty}{\infty}\end{array}$ & 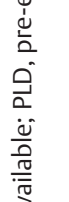 \\
\hline 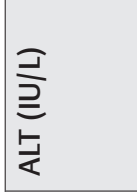 & 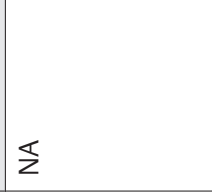 & 乏 & $\begin{array}{l}m \\
o \\
\sigma \\
+1 \\
m \\
\infty \\
\infty \\
\sim\end{array}$ & 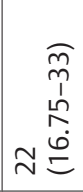 & 穴 & \begin{tabular}{l}
$\mathbb{N}$ \\
\multirow{1}{*}{}
\end{tabular} & 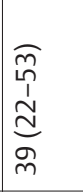 & $\begin{array}{l}a \\
\text { on } \\
1 \\
\frac{1}{d} \\
\text { m } \\
m\end{array}$ & 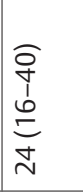 & 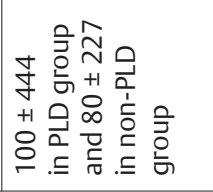 & 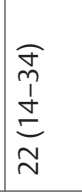 & $\begin{array}{l}\widehat{\bar{m}} \\
1 \\
\stackrel{n}{=} \\
\bar{v}\end{array}$ & 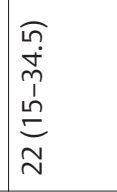 & $\frac{n}{\frac{n}{7}}$ & $\begin{array}{l}\dot{\Sigma} \\
\dot{u} \\
\dot{u}\end{array}$ \\
\hline 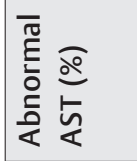 & $\begin{array}{l}\stackrel{\circ}{\tilde{N}} \\
\stackrel{N}{N}\end{array}$ & テ & 乏 & $\Sigma$ & $\Sigma$ & $\stackrel{\pi}{\check{r}}$ & $\stackrel{\leftrightarrow n}{m}$ & $\hat{m}$ & $\Sigma$ & 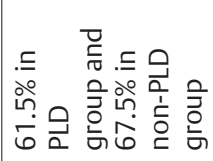 & $\bar{\emptyset}$ & $\begin{array}{l}\underset{\sim}{\infty} \\
\stackrel{\infty}{\sim}\end{array}$ & $\Sigma$ & $\stackrel{0}{\dot{N}}$ & $\stackrel{2}{\stackrel{2}{ \pm}}$ \\
\hline 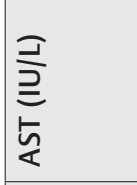 & $\Sigma$ & 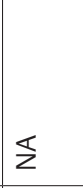 & $\begin{array}{l}\bar{m} \\
\dot{m} \\
+1 \\
\stackrel{N}{N}\end{array}$ & 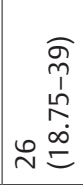 & $\infty$ & 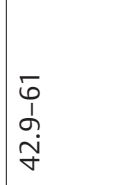 & 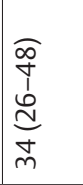 & 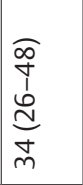 & 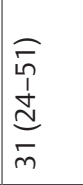 & 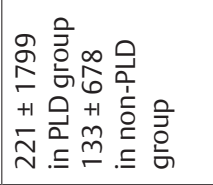 & 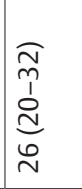 & 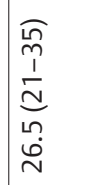 & 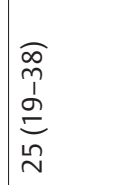 & $\begin{array}{l}\frac{0}{0} \\
\frac{1}{m} \\
\frac{m}{m}\end{array}$ & 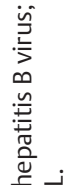 \\
\hline 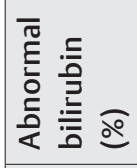 & $\stackrel{\llcorner}{\circ}$ & $\Sigma$ & $\Sigma$ & $\Sigma$ & $\Sigma$ & 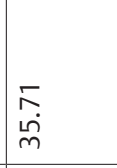 & $\stackrel{\infty}{\sim}$ & $\S$ & $\Sigma$ & 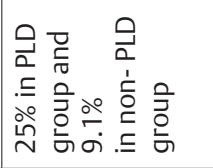 & $\S$ & $\begin{array}{l}\stackrel{\sigma}{\dot{N}} \\
\underset{\sim}{v}\end{array}$ & $\Sigma$ & $\overline{6}$ & 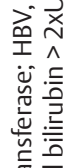 \\
\hline 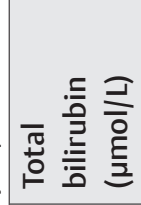 & $\Sigma$ & $\S$ & $\begin{array}{l}0 \\
\dot{m} \\
+1 \\
\tilde{n} \\
\tilde{i}\end{array}$ & 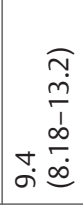 & $\Sigma$ & $\begin{array}{l}0 \\
\infty \\
\stackrel{\infty}{+} \\
\vdots \\
\infty \\
\infty \\
\infty\end{array}$ & 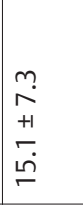 & 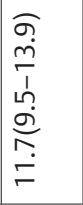 & $\begin{array}{l}a \\
\dot{\sigma} \\
\mp \\
+ \\
+\infty \\
\infty \\
\infty \\
\sigma \\
0\end{array}$ & 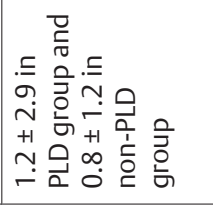 & $\Sigma$ & 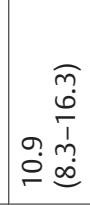 & 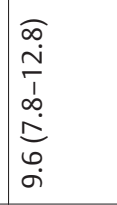 & $\begin{array}{l}0 \\
\dot{I} \\
I \\
\grave{N}\end{array}$ & 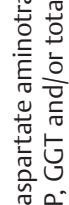 \\
\hline 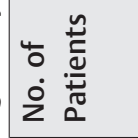 & g. & $\stackrel{ \pm}{\sim}$ & ‡ & भ & - & $\stackrel{ }{1}$ & જ & 广 & $\stackrel{\infty}{m}$ & 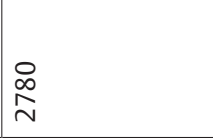 & $\widetilde{\sigma}$ & $\hat{\gamma}$ & $\stackrel{\cong}{\beth}$ & $\stackrel{\infty}{+}$ & 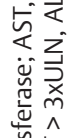 \\
\hline 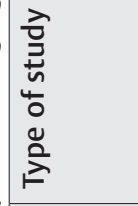 & 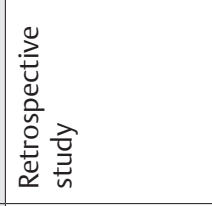 & 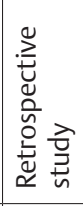 & 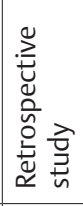 & 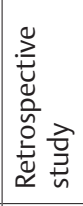 & 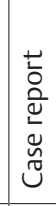 & 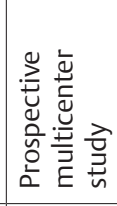 & 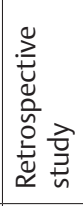 & 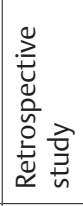 & 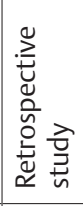 & 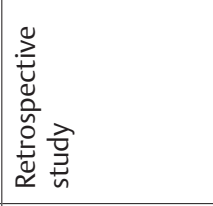 & 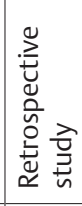 & 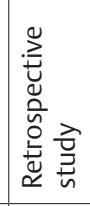 & 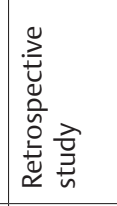 & 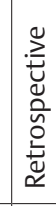 & 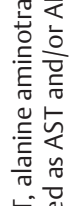 \\
\hline 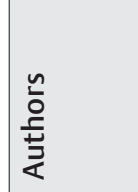 & 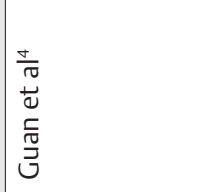 & 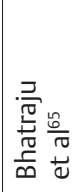 & 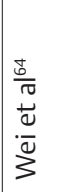 & 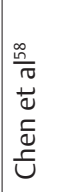 & 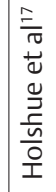 & $\begin{array}{l}\frac{\eta}{\pi} \\
\frac{\pi}{\pi} \\
\frac{\pi}{0} \\
\tilde{0}\end{array}$ & $\mid \begin{array}{l}\frac{\pi}{\pi} \\
\frac{\pi}{d} \\
\frac{\pi}{d} \\
\frac{d}{U}\end{array}$ & 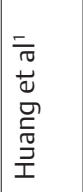 & 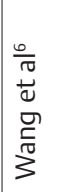 & $\begin{array}{l}\frac{5}{\pi} \\
\frac{5}{0} \\
\frac{5}{0} \\
\vdots \bar{n}\end{array}$ & $\begin{array}{l}\frac{0}{\pi} \\
\frac{0}{U} \\
\vec{x}\end{array}$ & 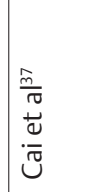 & 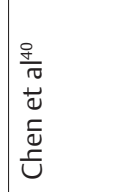 & 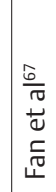 & 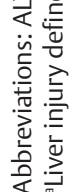 \\
\hline
\end{tabular}


Table 3 Drugs used for treatment in COVID-19, their mechanism of action, and GI side effects

\begin{tabular}{|c|c|c|c|c|}
\hline Treatment & Dose & Route & Class of drug & GI/Liver side effects \\
\hline Remdesivir & $\begin{array}{l}200 \mathrm{mg} \text { stat followed by } 100 \mathrm{mg} \\
\text { OD for } 10 \text { days }\end{array}$ & Intravenous & Antiviral & $\begin{array}{l}\text { Nausea, vomiting, elevated } \\
\text { transaminases }\end{array}$ \\
\hline Lopinavir/Ritonavir & $400 \mathrm{mg} / 100 \mathrm{mg}$ BD & Oral & Antiviral & $\begin{array}{l}\text { Deranged liver enzymes (not shown to } \\
\text { be clinically significant in a } \mathrm{RCT}^{68} \text { ) }\end{array}$ \\
\hline Hydroxychloroquine & $\begin{array}{l}800 \mathrm{mg} \text { stat followed by } 400 \mathrm{mg} \\
\text { daily }\end{array}$ & Oral & Antimalarial & $\begin{array}{l}\text { Nausea, vomiting, weight loss, } \\
\text { abdominal pain }\end{array}$ \\
\hline Chloroquine & $500 \mathrm{mg}$ BD & Oral & Antimalarial & $\begin{array}{l}\text { Elevated transaminases, anorexia, } \\
\text { nausea, vomiting, diarrhea, abdominal } \\
\text { cramps }\end{array}$ \\
\hline Azithromycin & $500 \mathrm{mg}$ OD & Oral & Antibacterial & $\begin{array}{l}\text { Diarrhea, nausea, vomiting, pain } \\
\text { abdomen }\end{array}$ \\
\hline Tocilizumab & $8 \mathrm{mg} / \mathrm{kg}$ IV single dose & Intravenous & $\begin{array}{l}\text { IL-6 receptor } \\
\text { antagonist }\end{array}$ & $\begin{array}{l}\text { Elevated transaminases, bowel } \\
\text { perforation, pancreatitis, abdominal } \\
\text { pain, reactivation of chronic hepatitis B }\end{array}$ \\
\hline Favipiravir & $\begin{array}{l}1000-1600 \mathrm{mg} \text { on first day } \\
\text { followed by } 400-800 \mathrm{mg} \text { BD for } \\
4-13 \text { days }\end{array}$ & Oral & Antiviral & Not reported \\
\hline Ivermectin & $\begin{array}{l}200 \mu \mathrm{g} / \mathrm{kg} \text { body weight single } \\
\text { dose }\end{array}$ & Oral & Antiparasitic & Nausea, vomiting, diarrhea \\
\hline
\end{tabular}

Abbreviations: Gl, gastrointestinal; RCT, randomized controlled trial.

The major expression is seen in cholangiocytes $(\sim 59.7 \%)^{29}$; however, injury to bile duct cells is not clinically evident, as ALP remains largely normal in patients infected with COVID-19. A recent study, however, showed elevated GGT in $54 \%$ of patients, suggesting injury to bile duct cells. ${ }^{31}$ Along with cholangiocytes, TMPRSS2 is also expressed in periportal liver sinusoidal endothelial cells and erythroid cells in liver, suggesting a pathologic basis for infection of liver by SARS$\mathrm{CoV}-2 .{ }^{30}$ In an autopsy series of 22 patients, ${ }^{32}$ low-levels of viral RNA were detectable in liver tissues. Viral particles have also been shown to be abundant in the cytoplasm of infected hepatocytes. ${ }^{33}$

\section{Inflammatory Cytokines}

Another contributor to liver injury is an inflammatory cytokine storm. Lymphopenia, commonly seen in these patients, leads to decreased inhibition of the innate immune response. As a result, Interleukin-6 (IL-6), IL-10, IL-12, and IFN- $\gamma$ are increased in circulation, which causes multiorgan dysfunction, including involvement of liver. ${ }^{34}$ The presence of lymphopenia and elevated CRP is independently associated with liver injury, ${ }^{35}$ which highlights the plausible role of cytokine storm in inciting liver dysfunction. Another interesting finding seen on immunohistochemistry of postmortem liver biopsies was the presence of increase CD68+ cells in hepatic sinusoid which is suggestive of Kupffer cell activation. ${ }^{33}$

\section{Ischemic Hepatitis and Drug Toxicity}

Ischemic hepatitis does not seem to be a major contributory factor in the elevation of transaminases as AST and ALT are only rarely elevated to $>5 \mathrm{x}$ ULN in patients with COVID$19 .{ }^{36}$ Also, features of ischemic hepatitis are not seen on liver biopsy. ${ }^{33}$ Various drugs used to treat COVID-19 can also lead to drug-induced liver injury (-Table 3 ). However, no concrete data are available regarding a plausible cause and effect relationship.

\section{Histopathological Findings in the Liver}

Theavailableliverbiopsyfindingsfrom patientswithCOVID-19 are limited. One patient showed mild vesicular steatosis with watery degeneration of hepatocytes and sinusoidal inflammation, ${ }^{37}$ while another patient showed moderate vesicular steatosis with mild lobular and portal activity. ${ }^{38} \mathrm{~A}$ case series of four patients described mild sinusoidal dilatation and lobular lymphocytic inflammation with no evidence of steatosis, and one of the patients showing patchy hepatocyte necrosis. ${ }^{39}$ A recent study ${ }^{33}$ also demonstrated mitochondrial swelling, endoplasmic reticulum dilatation, and a decrease in glycogen granule reserve of hepatocytes with massive hepatic apoptosis.

\section{COVID-19 and Chronic Liver Disease}

The prevalence of chronic liver disease among patients infected with COVID-19 is 2 to $11 \%$, as shown in -Table 2 . The prevalence of hepatitis $B$ virus (HBV) infection in patients with COVID-19 is approximately $12 \%{ }^{40}$ The patients with HBV infection had a higher prevalence of cirrhosis, elevated bilirubin ( $p=0.039$ and 0.0178 , respectively), more severe disease ( $46.7 \%$ vs. $2.8 \%$ ), and high mortality ( $13.3 \%$ vs. $2.8 \%$ ). The burden of alcohol-related liver disease was estimated to be around 30\% in hospitalized patients with COVID-19 in one series. $^{41}$

In another series, ${ }^{42}$ 2,780 COVID-19 patients were analyzed, out of which $9 \%$ had pre-existing chronic liver disease. The most common chronic liver disease coexisting with COVID-19 was NASH, seen in $42 \%$ of patients with pre-existing liver disease. ${ }^{42}$ Cirrhosis was seen in $1.8 \%$ cases, and 
patients with pre-existing liver disease and cirrhosis had a high-risk of mortality. Increased disease progression, higher likelihood of abnormal LFT, and longer viral shedding time of approximately 5 days in patients with nonalcoholic fatty liver disease NAFLD have also been reported. ${ }^{43}$

In another study describing the clinical course of COVID-19 in patients with autoimmune hepatitis (AIH) on immunosuppression, it was found that $70 \%$ of patients with $\mathrm{AIH}$ were asymptomatic. ${ }^{44}$ Respiratory symptoms were present in $26 \%$ cases, and they were classified as suspected cases since testing was not conducted. Only $3 \%$ of patients were confirmed COVID-19 cases. This suggests that the incidence of COVID19 in patients with $\mathrm{AIH}$ is similar to that seen in the general population.

\section{Liver Transplantation and COVID-19}

The number of liver transplants performed in the past 2 to 3 months has declined drastically in various transplant centers across the globe. The available data from Italy and the USA have shown that organ procurement for transplant had decreased by approximately $25 \%$ in the first month of the outbreak $^{45,46}$; however, the incidence of infection was low in liver transplant recipients. ${ }^{47}$ High mortality (23\%) has been reported in liver transplant recipients due to COVID-1941; hence, liver transplantation should only be done for emergency indications. Since there are concerns regarding donor and recipient safety, the Liver Transplant Society of India (LTSI) and National Organ and Tissue Transplant Organization (NOTTO) have recommended that elective transplants be deferred. ${ }^{48}$ They recommend that only transplants for acute liver failure and ACLF should be performed after donor and recipient testing and proceed only if COVID testing is negative. ${ }^{49}$

The management of COVID-19 in the posttransplant patient is also a challenge due to the interplay of immunosuppression, drug-induced liver injury, and direct cytopathic effects of SARS-CoV-2. A recent case report ${ }^{50}$ describes COVID-19 infection in a liver transplant recipient on postoperative day 19 , which was successfully managed while continuing tacrolimus and glucocorticoids. However, another report describes a 59-year-old patient,,11 who underwent a liver transplant in 2017 for decompensated HBV cirrhosis with hepatocellular carcinoma and succumbed to COVID-19 with nosocomial sepsis and multiorgan failure on day 45 of hospital admission. However, more data are required to identify the risk factors for COVID-related mortality in liver transplant recipients and guide immunosuppressive regimens and management after liver transplant.

\section{Gastrointestinal Endoscopy in the COVID-19 Era}

Gastrointestinal endoscopy is an aerosol-generating procedure, and given the high prevalence of SARS-CoV-2 viral RNA in feces of COVID-19 infected patients, it appears necessary to consider GI secretions as infective and capable of transmitting the virus during endoscopic procedures. Hence, it is necessary to take all necessary preventive measures to prevent the spread of disease to healthcare workers (HCWs).

Endoscopic procedures should be divided into emergency, urgent, and routine-based on their urgency. The current joint guidelines from Society of Gastrointestinal Endoscopy of India (SGEI), Indian Society of Gastroenterology (ISG), and Indian National Association for the Study of the Liver (INASL) state that only emergency and urgent endoscopy procedures be performed and elective endoscopy be postponed till the current threat of COVID-19 persists. ${ }^{52}$ All patients scheduled for endoscopy should be screened for fever, cough, shortness of breath, diarrhea, abdominal pain, nausea/vomiting, history of travel to or residence in a high-transmission zone of COVID-19, or contact with confirmed COVID-19 case. They should be assessed regarding the urgency of endoscopy, and elective procedures should be postponed. Based on the presence of symptoms or history of travel to or contact with a COVID-19 positive case, patients can be divided into a lowrisk group, intermediate-risk group, and high-risk group. The low-risk patients do not have any symptoms of COVID-19 or history of travel to an area of high-transmission zone or history of contact with a confirmed COVID-19 patient. The high-risk patients have at least one symptom, and either have a history of contact with COVID-19 case or history of travel to or stay in high-transmission zone. All those not fitting into either of these categories are classified as an intermediate-risk group. All high-risk patients should be tested for COVID-19 by RT-PCR. The endoscopy room should be a negative pressure room, have minimum and experienced staff, and all team members should wear personal protective equipment (PPE) including gloves, gown/plastic apron, N-95 mask and face shield. In all high-risk procedures, donning and doffing of PPE should follow standard protocol.

Although viral RNA has been documented in saliva and feces, whether it is infective is a question of debate, as data from Italy suggest that only one patient out of 851 who underwent endoscopy developed COVID-1953 and no HCW who came in contact with this patient developed respiratory symptoms. Although this was a retrospective study, it might suggest that endoscopy may be a low-risk procedure for transmission if proper precautions are followed.

\section{Conclusion}

COVID-19 is caused by a novel coronavirus, and data regarding the epidemiology, symptomatology, and transmission are rapidly evolving. As the outbreak is progressing, more and more cases with nonrespiratory symptoms and signs are being recognized, with liver and GI involvement being common. As viral RNA shedding has been documented in saliva and feces of infected patients, it becomes prudent for gastroenterologists and hepatologists to identify these symptoms early and take necessary precautions in performing diagnostic and therapeutic procedures in these patients. Although the quality of evidence is low, various gastrointestinal and hepatology associations have issued guidelines for management, which should be followed strictly until further data are available. 


\section{Conflict of Interest}

None declared.

\section{References}

1 Huang C, Wang Y, Li X, et al. Clinical features of patients infected with 2019 novel coronavirus in Wuhan, China. Lancet 2020;395(10223):497-506

2 Lu R, Zhao X, Li J, et al. Genomic characterisation and epidemiology of 2019 novel coronavirus: implications for virus origins and receptor binding. Lancet 2020;395(10224):565-574

3 WHO. COVID-19 Dashboard. Available at: https://covid19. who.int. Accessed June 5, 2020

4 Guan WJ, Ni ZY, Hu Y, et al; China Medical Treatment Expert Group for Covid-19. Clinical characteristics of Coronavirus Disease 2019 in China. N Engl J Med 2020;382(18):1708-1720

5 Fang D, Ma J, Guan J., et al. Manifestations of digestive system in hospitalized patients with novel coronavirus pneumonia in Wuhan, China: a single-center, descriptive study. Chin J Dig 2020. Doi 10.3760/cma.j.issn.0254-1432.2020.0005

6 Wang D, Hu B, Hu C, et al. Clinical characteristics of 138 hospitalized patients with 2019 novel Coronavirus-infected pneumonia in Wuhan, China. JAMA 2020. Doi: 10.1001/ jama.2020.1585

7 Sultan S, Altayar O, Siddique SM, et al; AGA. AGA Institute rapid review of the GI and liver manifestations of COVID-19, meta-analysis of international data, and recommendations for the consultative management of patients with COVID-19. Gastroenterology 2020. Doi: S0016-5085(20)30593-X

8 Siegel A, Chang PJ, Jarou ZJ, et al. Lung Base findings of Coronavirus Disease (COVID-19) on abdominal CT in patients with predominant Gastrointestinal symptoms. Am J Roentgenol 2020. Doi: 10.2214/AJR.20.23232

9 Luo S, Zhang X, Xu H. Don't overlook digestive symptoms in patients with 2019 novel Coronavirus disease (COVID-19) Clin Gastroenterol Hepatol 2020;18(7):1636-1637

10 Henry BM, de Oliveira MHS, Benoit J, Lippi G. Gastrointestinal symptoms associated with severity of coronavirus disease 2019 (COVID-19): a pooled analysis. Intern Emerg Med 2020. Doi: $10.1007 /$ s11739-020-02329-9

11 Tian Y, Rong L, Nian W, He Y. Review article: gastrointestinal features in COVID-19 and the possibility of faecal transmission. Aliment Pharmacol Ther 2020;51(9):843-851

12 Nobel YR, Phipps M, Zucker J, et al. Gastrointestinal Symptoms and COVID-19: Case-Control Study from the United States. Gastroenterology 2020. Doi: 10.1053/j.gastro.2020.04.017

13 Cui J, Li F, Shi Z-L. Origin and evolution of pathogenic coronaviruses. Nat Rev Microbiol 2019;17(3):181-192

14 Hoffmann M, Kleine-Weber H, Schroeder S, et al. SARS-CoV-2 cell entry depends on ACE2 and TMPRSS2 and is blocked by a clinically proven protease inhibitor. Cell2020. Doi: 10.1016/j. cell.2020.02.052

15 Hashimoto T, Perlot T, Rehman A, et al. ACE2 links amino acid malnutrition to microbial ecology and intestinal inflammation. Nature 2012;487(7408):477-481

16 Xiao F, Tang M, Zheng X, Liu Y, Li X, Shan H, Evidence for gastrointestinal infection of SARS-CoV-2. Gastroenterology2020. Doi: 10.1053/j.gastro.2020.02.055

17 Holshue ML, DeBolt C, Lindquist S, et al; Washington State 2019-nCoV Case Investigation Team. First case of 2019 Novel Coronavirus in the United States. N Engl J Med 2020;382(10): 929-936

18 To KK, Tsang OTY, Chik-Yan Yip C, et al. Consistent detection of 2019 novel coronavirus in saliva. Clin Infect Dis 2020. Doi 10.1093/cid/ciaa149

19 Liu Q, Wang R, Qu G, et al. Macroscopic autopsy findings in a patient with COVID-19. J Forensic Med 2020;36:1-3
20 National Health Commission of the People's Republic of China. Guidance on coronavirus disease (COVID-19), China (Vision 7) (in Chinese). Available at: http://www.gov.cn/zhengce/zhengceku/2020-03/04/content_54867 05.htm. Accessed June 5, 2020

21 Barton LM, Duval EJ, Stroberg E, Ghosh S, Mukhopadhyay S. COVID-19 autopsies, Oklahoma, USA. Am J Clin Pathol 2020;153(6):725-733

22 Qi X, Liu C, Jiang Z, et al. Multicenter analysis of clinical characteristics and outcome of COVID-19 patients with liver injury. J Hepatol 2020. Doi: 10.1016/j.jhep.2020.04.010

23 Chen N, Zhou M, Dong X, et al. Epidemiological and clinical characteristics of 99 cases of 2019 novel coronavirus pneumonia in Wuhan, China: a descriptive study. Lancet 2020;395(10223):507-513

24 Lei F, Liu YM, Zhou F, et al. Longitudinal association between markers of liver injury and mortality in COVID-19 in China. Hepatology 2020. Doi: 10.1002/hep.31301

25 Jin X, Lian JS, Hu JH, et al. Epidemiological, clinical and virological characteristics of 74 cases of coronavirus-infected disease 2019 (COVID-19) with gastrointestinal symptoms. Gut 2020;69(6):1002-1009

26 Weber S, Mayerle J, Irlbeck M, Gerbes AL. Severe liver failure during SARS-CoV-2 infection. Gut 2020. Doi: 10.1136/ gutjnl-2020-321350

27 Qiu H, Wander P, Bernstein D, Satapathy SK. Acute on chronic liver failure from novel severe acute respiratory syndrome coronavirus 2 (SARS-CoV-2) Liver Int 2020;00:1-4

28 Große K, Kramer M, Trautwein C, Bruns T, SARS - CoV - 2 as an extrahepatic precipitator of acute - on - chronic liver failure. Liver Int 2020. Doi: 10.1111/liv.14540

29 Pirola CJ, Sookoian S. SARS-CoV-2 virus and liver expression of host receptors: putative mechanisms of liver involvement in COVID-19. Liver Int 2020. Doi 10.1111/liv.14500

30 Chai X, Hu L, Zhang Y, et al. Specific ACE2 expression in cholangiocytes may cause liver damage after2019;-nCoV infection. bioRxiv 2020. Doi: 10.1101/2020.02.03.931766

31 Zhang C, Shi L, Wang FS. Liver injury in COVID-19: management and challenges. Lancet Gastroenterol Hepatol 2020;5(5): 428-430

32 Puelles VG, Lütgehetmann $M$, Lindenmeyer MT, et al. Multiorgan and renal tropism of SARS-CoV-2. N Engl J Med 2020. Doi: 10.1056/NEJMc2011400

33 Wang Y, Liu S, Liu H, et al. SARS-CoV-2 infection of the liver directly contributes to hepatic impairment in patients with COVID-19. J Hepatol 2020. Doi: 10.1016/j.jhep.2020.05.002

34 Liu J, Li S, Liu J, et al. Longitudinal characteristics of lymphocyte responses and cytokine profiles in the peripheral blood of SARS-CoV-2 infected patients. medRxiv 2020. Doi: 10.1016/j. ebiom.2020.102763

35 Lu L, Shuang L, Manman X, et al. Risk factors related to hepatic injury in patients with corona virus disease. medRxiv 2020. Doi: 10.1101/2020.02.28.20028514

36 Bangash MN, Patel J, Parekh D, COVID-19 and the liver: little cause for concern. The Lancet Gastroenterology \& Hepatology 2020;5(6)-P529-530

37 Cai Q, Huang D, Yu H. COVID-19: Abnormal liver function tests. J Hepatol 2020. Doi 10.1016/j.jhep.2020.04.006

38 Xu Z, Shi L, Wang Y, et al. Pathological findings of COVID-19 associated with acute respiratory distress syndrome. Lancet Respir Med 2020;8(4):420-422

39 Tian S, Xiong Y, Liu H, et al. Pathological study of the 2019 novel coronavirus disease (COVID-19) through postmortem core biopsies. Mod Pathol 2020. Doi: 10.1038/s41379-020-0536-X

40 Chen X, Jiang Q, Ma Z et al. Clinical characteristics of hospitalized patients with SARS-CoV-2 and hepatitis B virus co-infection. medRxiv 2020. Doi: 10.1101/2020.03.23.20040733 
41 Surveillance Epidemiology of Coronavirus (COVID-19) Under Research Exclusion April 6, 2020

42 Singh S, Khan A. Clinical characteristics and outcomes of COVID-19 among patients with pre-existing liver disease in United States: a multi-center research network study. Gastroenterology 2020. Doi: 10.1053/j.gastro.2020.04.064

43 Ji D, Qin E, Xu J, et al. Non-alcoholic fatty liver diseases in patients with COVID-19: A retrospective study. J Hepatol 2020. Doi: 10.1016/j.jhep.2020.03.044

44 Di Giorgio A, Nicastro E, Speziani C, et al. Health status of patients with autoimmune liver disease during SARS-CoV-2 outbreak in northern Italy. J Hepatol 2020. Doi: 10.1016/j. jhep.2020.05.008

45 Angelico R, Trapani S, Manzia TM, Lombardini L, Tisone G, Cardillo M. The COVID-19 outbreak in Italy: initial implications for organ transplantation programs. Am J Transplant 2020. Doi: $10.1111 /$ ajt.15904

46 Agopian V, Verna E, Goldberg D. Changes in liver transplant center practice in response to COVID-19: Unmasking dramatic center-level variability. Liver Transpl 2020. Doi: 10.1002/ lt.25789

47 Lauterio A, De Carlis R, Belli L, Fumagalli R, De Carlis L. How to guarantee liver transplantation in the north of Italy during the COVID-19 pandemic: a sound transplant protection strategy. Transpl Int 2020. Doi: 10.1111/tri.13633

48 National Transplant Specific Guidelines for COVID-19. 1754068/2020/NOTTO- DGHS; Annexure-A

49 Saigal S, Gupta S, Sudhindran S, et al. Liver transplantation and COVID-19 (Coronavirus) infection: guidelines of the Liver Transplant Society of India (LTSI). Hepatol Int 2020. Doi: $10.1007 / \mathrm{s} 12072-020-10041-1$

50 Qin J, Wang H, Qin X, et al. Perioperative presentation of COVID-19 disease in a liver transplant recipient. Hepatology 2020. Doi: 10.1002/hep.31257

51 Huang JF, Zheng KI, George J, et al. Fatal outcome in a liver transplant recipient with COVID-19. Am J Transplant 2020;00:1-4

52 Philip M, Lakhtakia S, Aggarwal R, Madan K, Saraswat V, Makharia G. Joint guidance from SGEI, ISG and INASL for gastroenterologists and gastrointestinal endoscopists on the prevention, care, and management of patients with COVID-19. J Clin Exp Hepatol 2020;10(3):266-270

53 Repici A, Aragona G, Cengia G, et al; ITALIAN GI-COVID19 Working Group. Low risk of covid-19 transmission in GI endoscopy. Gut 2020;0:1-3

54 Jiang X, Luo M, Zou Z, et al. Asymptomatic SARS-CoV-2 infected case with viral detection positive in stool but negative in nasopharyngeal samples lasts for 42 days. J Med Virol 2020. Doi: 10.1002/jmv.25941
55 Siew C, Ng, Francis, KL, Chan, Paul KS, Chan. Screening FMT donors during the COVID-19 pandemic: a protocol for SARSCoV-2 viral quantification. Lancet Gastroenterol Hepatol 2020. Doi: 10.1016/S2468-1253(20)30124-2

$56 \mathrm{Wu}$ J, Liu J, Li S, et al. Detection and analysis of nucleic acid in various biological samples of COVID-19 patients. Travel Med Infect Dis 2020. Doi:10.1016/j.tmaid.2020.101673

57 Wu Y, Guo C, Tang L, et al. Prolonged presence of SARS-CoV-2 viral RNA in faecal samples. Lancet Gastroenterol Hepatol 2020;5(5):434-435

58 Chen Y, Chen L, Deng Q et al. The presence of SARS-CoV-2 RNA in the feces of COVID-19 patients. J Med Virol 2020. Doi:10.1002/jmv.25825

59 Chen L, Lou J, Bai Y, Wang M. COVID-19 disease with positive fecal and negative pharyngeal and sputum viral tests. Am J Gastroenterol 2020;115(5):790

60 Zhang W, Du RH, Li B, et al. Molecular and serological investigation of 2019-nCoV infected patients: implication of multiple shedding routes. Emerg Microbes Infect 2020;9(1):386-389

61 Yun H, Sun Z, Wu J, Tang A, Hu M, Xiang Z. Laboratory data analysis of novel coronavirus (COVID-19) screening in 2510 patients. Clin Chim Acta 2020;507:94-97

62 Cheung KS, Hung IF, Chan PP, et al. Gastrointestinal manifestations of SARS-CoV-2 infection and virus load in fecal samples from the Hong Kong cohort and systematic review and meta-analysis. Gastroenterology 2020. Doi: 10.1053/j. gastro.2020.03.065

63 Zhang J, Wang S, Xue Y. Fecal specimen diagnosis 2019 novel coronavirus-infected pneumonia. J Med Virol 2020;92: 680-682

64 Wei X-S, Wang X, Niu Y-R, et al. Diarrhea is associated with prolonged symptoms and viral carriage in COVID-19. Clin Gastroenterol Hepatol 2020. Doi: 10.1016/j.cgh.2020.04.030

65 Bhatraju PK, Ghassemieh BJ, Nichols M, et al. Covid-19 in critically ill patients in the Seattle region - Case Series. N Engl J Med 2020;382(21):2012-2022

$66 \mathrm{Xu} \mathrm{X-W,} \mathrm{Wu} \mathrm{X-X,} \mathrm{Jiang} \mathrm{X-G,} \mathrm{et} \mathrm{al.} \mathrm{Clinical} \mathrm{findings} \mathrm{in} \mathrm{a} \mathrm{group}$ of patients infected with the 2019 novel coronavirus (SARSCov-2) outside of Wuhan, China: retrospective case series. BMJ 2020. Doi: $10.1136 /$ bmj.m792

67 Fan Z, Chen L, Li J, Cheng X, Jingmao Yang Tian C, Zhang Y, et al Clinical Gastroenterology and Hepatology. Clinical features of COVID-19-related liver damage. 2020;18(7)1561-1566

68 Cao B, Wang Y, Wn D, et al. A Trial of lopinavir-ritonavir in adults hospitalized with severe Covid-19. N Engl J Med 2020;382:1787-1799 